\title{
Serum Vitamin D, Folate and Fatty Acid Levels in Children with Autism Spectrum Disorders: A Systematic Review and Meta-Analysis
}

\author{
Maria Carmen Gallardo-Carrasco ${ }^{1}\left[\right.$. José Antonio Jiménez-Barbero ${ }^{2}\left[\right.$ - María del Mar Bravo-Pastor ${ }^{3}$. \\ David Martin-Castillo ${ }^{4} \cdot$ María Sánchez-Muñoz $^{1}$
}

Accepted: 18 October 2021 / Published online: 3 November 2021

(c) The Author(s) 2021

\begin{abstract}
Vitamin and fatty acid deficiency in children diagnosed with autism has been linked to the etiology and course of the disease but the results have been inconsistent. In our work, we present a narrative review, which includes 20 observational studies that provide data on the blood levels of vitamin D, folate, or fatty acids of children diagnosed with ASD (Autism Spectrum Disorder-AG group), and of a control group (children without this disorder-CG group). The main characteristics and results are presented in a summary table. Of the 20 above-mentioned studies, a meta-analysis of vitamin $\mathrm{D}$ and folate levels was carried out in 14 of them, with a total of 2269 children $(A G=1159, C G=1110)$. Vitamin D levels were lower in AG compared to CG: SMD, 95\% CI $=-0.83$ [-1.15, - 0.50]. In terms of folate levels, a total of 299 children $(\mathrm{AG}=148$, $\mathrm{CG}=151$ ) were analyzed, finding no significant differences with the control group: SMD, 95\% CI $=-0.16[-0.63,0.32]$. Only one study that provided data on fatty acids in children with ASD was included in the review although it was not possible to include it in the meta-analysis. We conclude that the nutritional status (vitamin and fatty acid levels) of patients diagnosed with ASD should be taken into account, as correct adjustment of these levels-may produce an improvement in the course of the disease and could also reduce the risk of its development.
\end{abstract}

Keywords Autism, autism spectrum disorders $\cdot$ Fatty acids $\cdot$ Vitamin D $\cdot$ Folic acid levels

José Antonio Jiménez-Barbero

joseantonio.jimenez1@um.es

Maria Carmen Gallardo-Carrasco

gallardocarrasco@gmail.com

María del Mar Bravo-Pastor

marpastorbravo@um.es

David Martin-Castillo

david.martin@um.es

María Sánchez-Muñoz

mariahoradada@hotmail.com

1 "Los Arcos" Mental Health Center, Paraje Torre Octavio, 54, PC: 30739 Pozo Aledo, Murcia, Spain

2 Department of Nursing, University of Murcia, Espinardo Campus, Building 23, PC: 30100 Murcia, Spain

3 Nursing Department, University School of Nursing of Cartagena, University of Murcia, , Campus de Cartagena, Paseo Alfonso XIII, 61, PC: 30203 Cartagena, Murcia, Spain

4 Department of Nursing, University of Murcia, Espinardo Campus, Building 23, PC: 30100 Murcia, Spain

\section{Introduction}

Autism is a neurodevelopmental disorder initially described by Kanner (1943), characterized by alterations in social and verbal communication, limited and repetitive interests, and currently considered a serious mental disorder (Lord et al., 2018).

The global prevalence trend is a marked rise (Kerub et al., 2021; Málaga et al., 2019; Tioleco et al., 2021), although it is worth noting the great variability between the data depending on the country of the study (Belinchón et al., 2010; Fuentes et al., 2020; Málaga et al., 2019; Marín et al., 2016; Matson \& Kozlowski, 2011; Nevison \& Parker, 2020). In the US, according to the latest update from the Center for Disease Control and Prevention (CDC), the prevalence of ASD (Autism Spectrum Disorder) was 18.5 per 1000 (1:54) 8-year-olds, and ASD was 4.3 times more prevalent among boys than among girls. This prevalence varied by birthplace, from 13.1 (Colorado) to 31.4 (New Jersey) (Maenner et al., 2020). However, in Spain, the prevalence ranges from $15.5 / 1000$ (3-4 years) to $10 / 1000$ (10-11 years), with a ratio 
of 1:64 (3-4 years) to 1:100 (10-11 years) (Morales-Hidalgo et al., 2018).

The etiology of ASD remains little known, although different areas might be related (Kheirouri \& Alizadeh, 2020). Thus, for example, the influence of genetics, inflammatory processes, autoimmune disorders, oxidative stress, neurotransmitters, and environmental factors has been demonstrated (Chen et al., 2021; Kinney et al., 2010; Thorsen et al., 2021). Similarly, prenatal and perinatal events, such as maternal infection, medication, and parents' age have been identified as possible risk factors for autism and have received considerable attention (Guo et al., 2020).

Different studies have reported the finding of differences in blood levels of vitamins $\left(\mathrm{D}_{2,3}\right.$ and $\left.\mathrm{B}_{9}\right)$ and fatty acids in children diagnosed with autism, compared to children without this disorder (Du et al., 2015). We note that vitamin D, ergocalciferol (D2) or colecalciferol (D3), is a fat-soluble vitamin present in the diet in limited amounts, which is mainly obtained from skin exposure to UVB radiation (De Luca, 2004). This differs from folate or B9, a water-soluble vitamin obtained through the diet. According to the indications of the "Endocrine Society", vitamin D deficiency is defined as a serum concentration of $25(\mathrm{OH}) \mathrm{D}<20 \mathrm{ng} /$ $\mathrm{mL}$, insufficiency as $21-29 \mathrm{ng} / \mathrm{ml}$, and sufficiency as at least $30 \mathrm{ng} / \mathrm{ml}$ for adequate health (Holick, 2017).

The relationship between vitamin D deficiency and ASD is not entirely clear, and there are numerous studies, some of them quite recent, that relate deficient levels of these biochemical parameters in children diagnosed with ASD, compared to children without neurodegenerative pathologies (Agostoni et al., 2017; Arastoo et al., 2018; Bala et al., 2016; Bozzatello et al., 2016; Eyles et al., 2013; Farid et al., 2016; Frye et al., 2018; Garipardic et al., 2017; Guo et al., 2019; Liu et al., 2016; Parletta et al., 2016).

In this sense, rigid behavior, as children with ASD usually pose difficulty accepting changes in food textures or incorporating new foods into their daily diet (Cermak et al., 2010; Esteban-Figuerola et al., 2019; Sánchez et al., 2015; Yule et al., 2020), and difficulty relating to others, as well as sharing outdoor spaces with others, thus hindering sun exposure (Liu et al., 2015; Wang et al., 2021), have been described as causes that justify the pattern of vitamin D deficiency in children with ASD. Other authors propose dysfunction in the gut microbiota and gut-microbiota-brain relationship (Ogbu et al., 2020), as well as a decrease in vitamin D receptors (Altun et al., 2018).

\section{The Present Study}

The bibliography review has shown evidence of the relationship between vitamin $\mathrm{D}$ and folate levels and the diagnosis of autism in children (Altun et al., 2018; Frye et al., 2018; Garipardic et al., 2017; Gong et al., 2014; Mazahery et al., 2016; Sun et al., 2016). In fact, a recent meta-analysis assessed the relationship between vitamin D levels and autism, including studies published through November 2019 (Wang et al., 2021). Our work, however, represents an update and extension of previous studies, as it includes studies published until December 2020 that analyze vitamin D levels, folate, and fatty acids in children diagnosed with ASD versus children without this diagnosis.

The main objective is therefore to study the differences in blood levels of vitamins and fatty acids between children diagnosed with ASD compared to children without this diagnosis. For this purpose, a meta-analysis of observational studies was carried out that established comparisons between the values obtained in children diagnosed with ASD compared to children without this disorder. In addition, a narrative review is offered which considered all the studies that met the inclusion criteria but did not provide sufficient data to perform the meta-analysis.

Following the PRISMA 2020 (Page et al., 2021) criteria for research question formulation, we drew on the following approach: Are there significant differences in the reported levels of vitamin $\mathrm{D}$, folate, and fatty acids in children diagnosed with ASD compared to children without this diagnosis?

\section{Method}

The protocol used in this systematic review follows the recommendations of (Page et al., 2021). Similarly, the methods used in the review were specified in advance and documented in a protocol, which is available online: (https:// www.crd.york.ac.uk/PROSPEROFILES/125932_PROTO COL_225905.pdf).

\section{Inclusion and Exclusion Criteria}

According to the research question posed, the studies were included in the review if they met the following criteria: (a) studies should compare blood levels of vitamin D and/ or fatty acids and/or folate in children diagnosed with ASD with the values in children without this disorder; (b) the participants' age was between 0 and 18 years; (c) studies should have been published between 2014 and 2020; (d) ASD was diagnosed using the Diagnostic and Statistical Manual of Mental Disorders, fifth edition DSM-V (APA, 2014); (e) the age of the control group must range from 0 to 18 years and they should not have any neurodevelopmental pathology.

The outcome measures included in the study were:

1. Vitamin D levels, 25-hydroxyvitamin D, or 25-OH vitamin D, obtained through blood sample and analyzed in 
the laboratory by chemiluminescence immunoassay and expressed as $\mathrm{ng} / \mathrm{ml}$.

2. Levels of folic acid, folate, folacin, pteroylmonoglutamic acid, or vitamin $\mathrm{B}_{9}$, obtained through blood sample and analyzed in the laboratory with an enzyme immunoassay technique (ELISA).

3. Fatty acid levels. The three main omega-3 fatty acids measured are: alpha-linolenic acid (ALA), eicosapentaenoic acid (EPA), and docosahexaenoic acid (DHA). Obtained through blood sample and analyzed in the laboratory by capillary gas chromatography, with flame ionization detection.

Exclusion criteria were: (a) articles that did not propose among their objectives the measurement of blood levels of vitamin $\mathrm{D}$, folate, and fatty acids in people diagnosed with ASD; (b) that the study population was over 18 years of age; (c) secondary studies (narrative or systematic reviews).

\section{Search Strategy}

A systematic search in the following electronic databases was conducted: Medline, Cochrane, Pubmed, PsycINFO, and Web of Science (WOS). The main descriptors used were: "25-OH" AND "cholecalciferol" OR "vitamin" AND “D3" OR "vitamin D3" AND “autistic disorder" OR "autism" OR "ASD". The last search was performed on January 10, 2021, in the WOS. The complete strategy used can be found in "Addendum 1 ".

The search was conducted by two independent researchers who made lists of potentially eligible articles. These lists were subsequently agreed upon, and any disagreements were resolved through the intervention of a third reviewer. To reduce unplanned duplication of comments and to provide transparency to the review process, as well as to minimize reporting bias (Booth et al., 2013), this study was recorded in PROSPERO (International Prospective Register of Ongoing Systematic Reviews) http://www.crd.york.ac.uk/prospero since its initiation; Registry No: CRD4225905.

\section{Selection of the Studies}

Study selection was carried out in two phases, following the indications of the PRISMA statement (Moher et al., 2015):

- In the first phase, two reviewers independently examined potentially eligible studies by reading titles and abstracts, following a previously made checklist, which included the selection criteria described in the protocol. Listings of preselected articles were subsequently agreed upon, resolving discrepancies by discussion.

- In the second phase, two reviewers independently read the full text of the studies preselected in the previous phase, again creating two lists of potentially eligible articles.
Disagreements were resolved through discussion, and a third reviewer was required to intervene when no consensus was reached. The complete texts of the accepted articles were carefully read, and their lists of bibliographic references were examined to identify possible relevant articles that had not been located in the initial search.

\section{Analysis of Bias Risk}

The selected studies were subsequently submitted to riskof-bias analysis, which was performed by two independent reviewers. The instrument used by these reviewers was the STROBE initiative statement for observational studies consisting of 22 items (Von Elm et al., 2007). 1 point per item was awarded, using the following criteria: 1 point if the item was fulfilled, 0.5 points if the item was partially fulfilled, and 0 points if the item was not fulfilled at all. The cut-off point for the eligibility of the studies was established at the mean value of each scale, that is, the article had to exceed $50 \%$ of items on the evaluation scale to be included in the systematic review. In cases where no consensus was found for the acceptability of an article, a third reviewer was consulted. Finally, interjudge reliability was calculated using intraclass correlation analysis.

\section{Tabulation and Data Analysis}

The studies included in our study were coded in an Excel database by the first author. The coding was reviewed by the second and third authors, and doubts were resolved through discussion among all the authors. Subsequently, summary tables were created in which the data of each selected study were recorded according to the following categories: date and country of study, research objective, size and age of sample used, study design, outcome measures, and significant results.

\section{Data Synthesis}

First, a narrative synthesis of the included studies was carried out, based on the outcome measures: folate levels, vitamin D, and fatty acid levels. An ad-hoc table was developed with the main characteristics and results (Table 1).

The studies that provided data on means and standard deviation were included in a meta-analysis. The effects of the studies were compared by estimating the standardized mean differences (SMD) in the score of vitamin D and folate levels among children diagnosed with ASD (ASD group; AG) and children who were not diagnosed (control group; CG). The differences between the $\mathrm{SG}$ and the $\mathrm{CG}$ for each comparison were grouped to obtain the estimation of the total effect. Statistical models of random effects were applied, considered to be more appropriate for the 


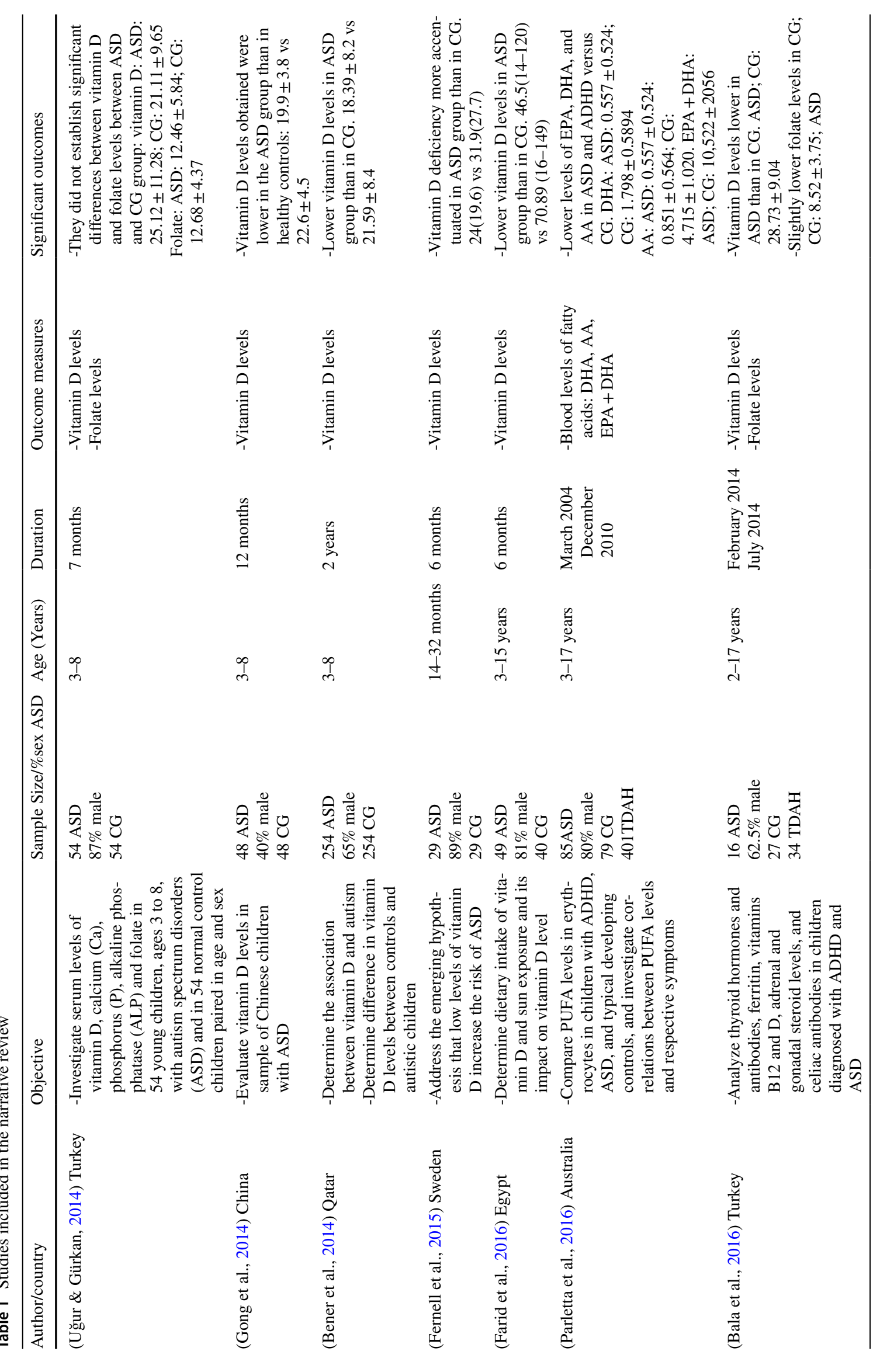




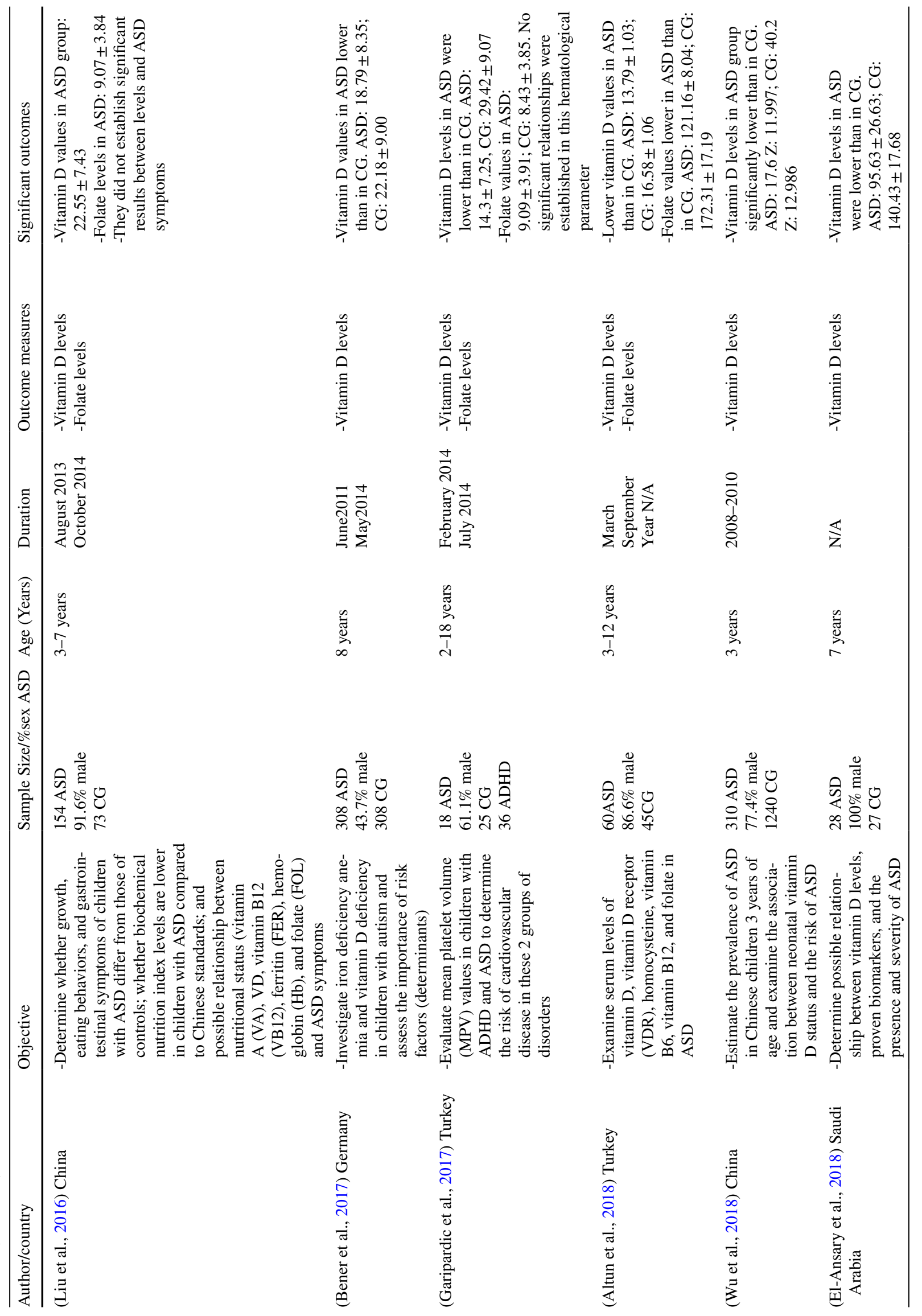




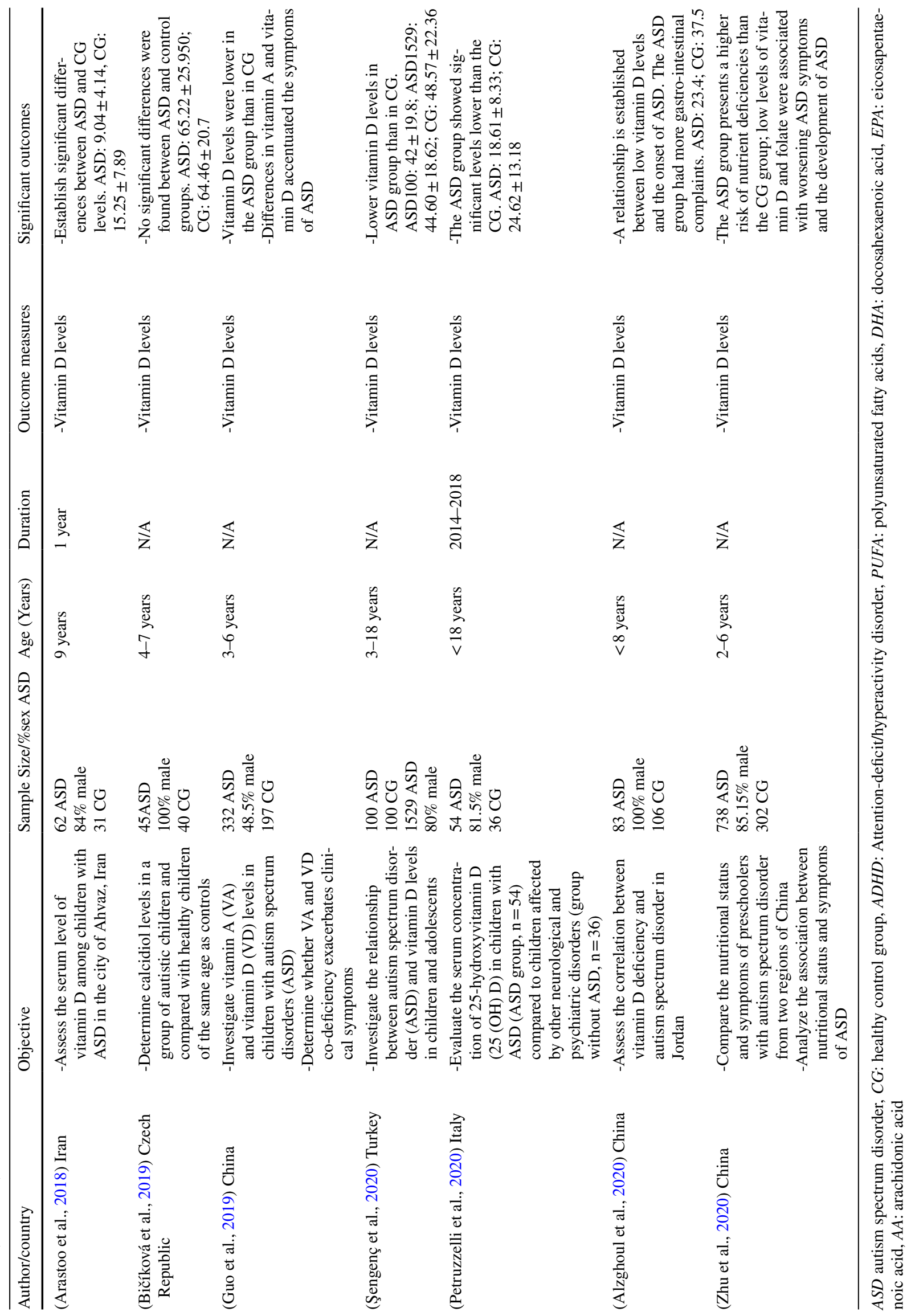


integration of the results of empirical studies due to the variability they usually present (Sánchez-Meca \& MarínMartínez, 2010), which was subsequently corroborated by the corresponding diagnoses of heterogeneity.

To determine the influence of each of the studies on the overall estimate of the effect, a sensitivity analysis was performed. For each comparison, the heterogeneity of the results was calculated using the chi-square test with a significance level of 0.05 , and the $\mathrm{I}^{2}$ index was also calculated. For cases where heterogeneity was significant, a subgroup analysis was carried out. Finally, we included in the analysis a study of publication bias to determine whether this could be a threat to the validity of the results of the meta-analysis. RevMan 5.3 was used for the calculations (Cochrane Center).

\section{Results}

As shown in Fig. 1, the electronic search initially located 4.715 publications, of which 63 were excluded because they were duplicate documents. After reading the title and abstract, 4559 studies were excluded as they did not respond to the objectives of the meta-analysis or to the research question formulated according to the PRISMA criteria. In the second phase of selection, after full-text reading, 52 articles were excluded for not meeting the inclusion criteria
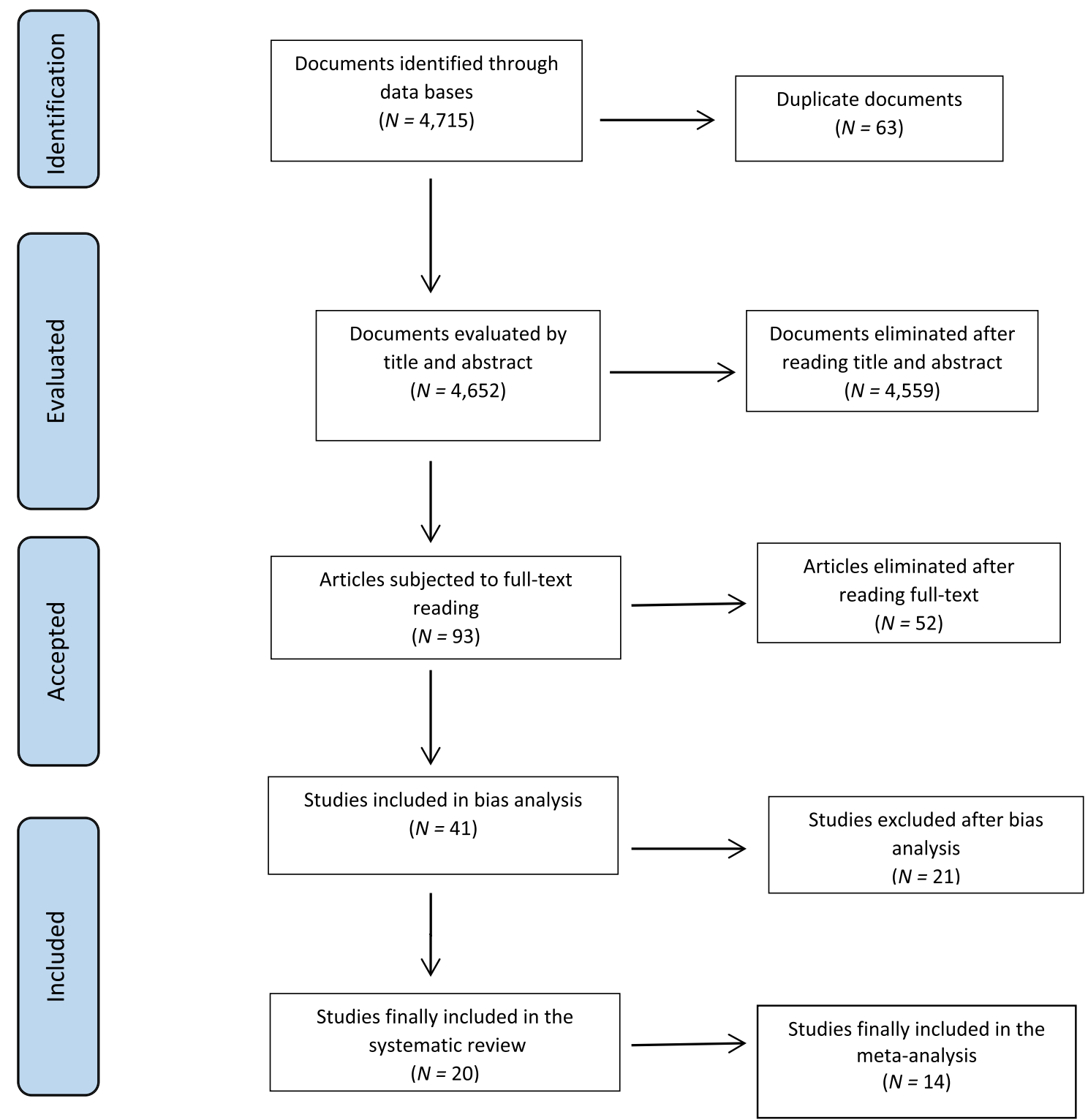

Fig. 1 The selection process following PRISMA guidelines (Page et al., 2021) 
established in the check-lists. Subsequently, after performing the risk-of-bias analysis, 21 studies were excluded for failing to meet the established methodological quality criteria. The scores given by each reviewer to each of the accepted studies, as well as the final score obtained by consensus, are available online, in a document annexed to the protocol.

Finally, 20 articles were included in the narrative review, all of which were quantitative observational studies. Except for one study that analyzed fatty acid (Parletta et al., 2016) levels, in the rest, measurements of vitamin D and folate levels were performed in a sample of children with ASD $(N=4171)$ and compared to a control group of healthy children or children not diagnosed as ASD $(N=2982)$. The intraclass correlation coefficient showed high interjudge reliability $(I C C=0.989, p<0.001)$.

Of the 20 selected studies, five provided incomplete data on or directly did not provide means and standard deviations (Farid et al., 2016; Guo et al., 2019; Liu et al., 2016; Wu et al., 2018; Zhu et al., 2020). The authors of these studies were contacted, but as we received no reply, we decided to exclude them from the meta-analysis. In addition, the study of Parletta et al. (2016) was excluded from the quantitative synthesis because it was the only one offering data on fatty acids. However, the 6 studies mentioned were included in the narrative synthesis due to the interest of their results. Finally, 14 of the reviewed studies that reported vitamin D and folate levels were included in the meta-analysis.

\section{Studies Included in the Narrative Review}

The 20 quantitative studies included were published between 2014 and 2020. The study sample has the following characteristics: the age range is between 0 and 18 years, the minimum and maximum sample sizes in the ASD group were 16 and 1529 , respectively, and in the control group, they were 25 and 1240. As for the sex of the sample, it was mostly male (Table 1) and the design of the studies was entirely quantitative and observational.

Three categories were established that correspond to the main objective of our study: vitamin D levels, folate levels, and blood levels of fatty acids.

\section{Vitamin D Levels}

Of the 20 studies included in the review, 19 papers provide data on vitamin D levels. As can be seen in Table 1, in 16 articles out of the total included in our work, vitamin D levels are shown to be lower in subjects belonging to the ASD group than in subjects in the control group (Altun et al., 2018; Alzghoul et al., 2020; Arastoo et al., 2018; Bala et al., 2016; Bener et al., 2014, 2017; El-Ansary et al., 2018; Farid et al., 2016; Fernell et al., 2015; Garipardic et al., 2017;
Gong et al., 2014; Guo et al., 2019; Petruzzelli et al., 2020; Şengenç et al., 2020; Wu et al., 2018; Zhu et al., 2020).

Moreover, three of the studies analyzed found no significant differences between the blood levels in the two groups (Bičíková et al., 2019; Liu et al., 2016; Uğur \& Gürkan, 2014).

\section{Folate Levels}

Blood levels of folate were measured in five of the selected studies (Altun et al., 2018; Bala et al., 2016; Garipardic et al., 2017; Liu et al., 2016; Uğur \& Gürkan, 2014). Altun et al. (2018) achieved significant results in terms of the ASD group's folate levels compared to the CG; lower blood levels were obtained in subjects in the ASD group than those of healthy control: ASD: $121.16 \pm 8.04$; Control:172.31 \pm 17.19 $(\mathrm{M} \pm \mathrm{SD})$.

The rest of the work about folate in the selected studies did not obtain significant results for this hematological parameter (Table 1).

\section{Fatty Acid Levels}

Only one study was included in this review that offered data on fatty acid levels and met methodological quality criteria (Parletta et al., 2016). In this study, in a sample of 85 children with ASD and 401 with Attention Deficit Hyperactivity Disorder (ADHD) compared to 79 of the control group, the authors analyzed the blood levels of DHA, AA, EPA + DHA, finding lower values in the ASD and ADHD group compared to the CG of healthy subjects (Table 1).

\section{Studies Included in the Meta-Analysis}

Meta-analysis was performed for vitamin D and folate levels of 14 of the previously selected studies (Altun et al., 2018; Alzghoul et al., 2020; Arastoo et al., 2018; Bala et al., 2016; Bener et al., 2014, 2017; Bičíková et al., 2019; El-Ansary et al., 2018; Fernell et al., 2015; Garipardic et al., 2017; Gong et al., 2014; Petruzzelli et al., 2020; Şengenç et al., 2020; Uğur \& Gürkan, 2014) to obtain an estimate of the average effect size for a $95 \%$ confidence interval. For heterogeneity analysis, the values of $\chi^{2}$ and the $I^{2}$ index were estimated.

\section{Vitamin D Levels}

The data of 2269 adolescents were available $(\mathrm{AG}=1159$, $\mathrm{CG}=1110$ ), included in the 14 complete trials that measured this variable. Vitamin D levels were lower in the group of children diagnosed with ASD compared to children in the CG: SMD, 95\% CI $=-0.83$ [-1.15, -0.50], with significant heterogeneity $\left(\chi^{2}=153.35, p=0.0001 ; I^{2}=92 \%\right)$. Given 


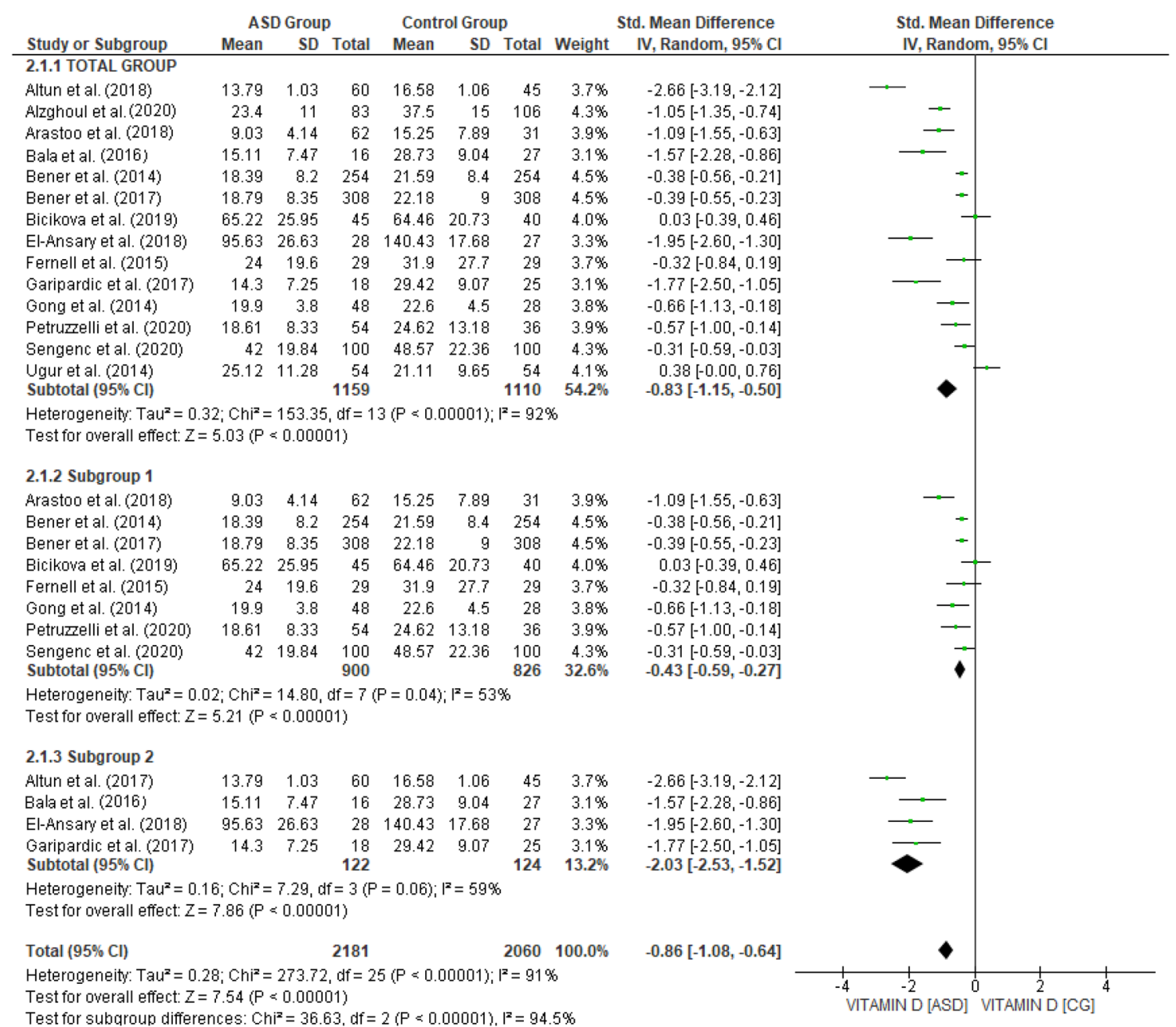

Fig. 2 Vitamin D levels ASD Group vs Control Group

the high heterogeneity observed, a subgroup analysis was performed based on the moderating variables, which did not explain the heterogeneity obtained. A sensitivity analysis was then performed, repeating the calculations by extracting the studies one at a time. This second analysis established two groups, included in Fig. 2.

The subgroup that included the highest number of studies ( 8 studies, $N=1726$; $\mathrm{SG}=900, \mathrm{CG}=826$ ) continued to show lower levels of vitamin $\mathrm{D}$ in children with ASD, although the average effect size remained small, SMD, 95\% CI $=-0.43$ [ $-0.59,-0.27]$, observing greater homogeneity between the different studies $\left(\chi^{2}=14.80, p=0.04 ; I^{2}=53 \%\right)$. The second subgroup (4 studies, $N=246$; $\mathrm{SG}=122 ; \mathrm{CG}=124$ ) maintained the trend observed in the previous analyses, although it had a larger mean effect size, SMD, 95\% CI $=-2.03[-2.53$, $-1.52]$, and the heterogeneity analysis was non-significant $\left(\chi^{2}=7.29, p=0.06 ; I^{2}=53 \%\right)$. Significant differences were observed between the two subgroups $\left(\chi^{2}=36.63\right.$, $p<0.001 ; I^{2}=94.5 \%$ ).

\section{Folate Levels}

As shown in Fig. 3, data on 299 children were available ( $\mathrm{SG}=148, \mathrm{CG}=151)$, included in 4 complete observational studies measuring this variable. Folate levels showed no significant differences in the group of children diagnosed with ASD compared to children in the CG: SMD, 95\% $\mathrm{CI}=-0.16[-0.63,0.32]$, with significant heterogeneity $\left(\chi^{2}=11.56, p=0.009 ; I^{2}=76 \%\right)$. Given the scarcity of studies that measured this variable and considering that no significant differences were observed between the two groups, no analyses were carried out to explain this heterogeneity. 


\begin{tabular}{|c|c|c|c|c|c|c|c|c|c|}
\hline \multirow[b]{2}{*}{ Study or Subgroup } & \multicolumn{3}{|c|}{ ASDGROUP } & \multicolumn{3}{|c|}{ CONTROL GROUP } & \multicolumn{2}{|r|}{ Std. Mean Difference } & \multirow{2}{*}{$\begin{array}{l}\text { Std. Mean Difference } \\
\text { IV, Random, } 95 \% \mathrm{Cl} \\
\end{array}$} \\
\hline & Mean & SD & Total & Mean & $S D$ & Total & Weight & IV, Random, $95 \% \mathrm{Cl}$ & \\
\hline Altun et al. (2017) & 12.116 & 8.04 & 60 & 17.2 & 3.11 & 45 & $27.7 \%$ & $-0.79[-1.19,-0.39]$ & $t$ \\
\hline Bala et al. (2016) & 9.17 & 3.96 & 16 & 8.52 & 3.75 & 27 & $21.8 \%$ & $0.17[-0.45,0.79]$ & \\
\hline Garipardic et al. (2017) & 9.09 & 3.91 & 18 & 8.43 & 3.85 & 25 & $22.1 \%$ & $0.17[-0.44,0.77]$ & \\
\hline Ugur et al. (2014) & 12.46 & 5.84 & 54 & 12.68 & 4.37 & 54 & $28.4 \%$ & $-0.04[-0.42,0.33]$ & \\
\hline Total $(95 \% \mathrm{Cl})$ & & & 148 & & & 151 & $100.0 \%$ & $-0.16[-0.63,0.32]$ & \\
\hline $\begin{array}{l}\text { Heterogeneity: } \text { Tau }^{2}=0 \text {. } \\
\text { Test for overall effect: } Z\end{array}$ & $\begin{array}{l}17 ; \mathrm{Chi}^{2}= \\
=0.65(\mathrm{P}\end{array}$ & & & $P=0.0$ & $1,1-$ & & & & $\begin{array}{ccc}-4 & -2 & 0 \\
& & 2\end{array}$ \\
\hline
\end{tabular}

Fig. 3 Folate levels ASD Group vs Control Group

\section{Analysis of Publication Bias}

To assess the bias potential for this study, a funnel plot was performed for each of the meta-analyses (Addendum 2). For both measures, the distribution was symmetrical, so there could be no publication bias that would compromise the interpretation of effect sizes found. However, it should be considered that there were few comparisons in the metaanalysis of folate levels, so this type of graph might not be appropriate to detect this possible bias.

\section{Discussion}

The main objective of this work was to study differences in blood levels of vitamins and fatty acids between children diagnosed with ASD compared to children without this diagnosis.

The results obtained in the meta-analysis showed statistically significant differences in the blood levels of vitamin $\mathrm{D}$, which were lower in children diagnosed with ASD than in the CG. The data obtained largely coincide with the meta-analysis of Wang et al. (2016), considered to be the first meta-analysis that investigated a possible relationship between low vitamin D levels and ASD. This work, which included 11 studies published between January 1980 and May 2015, with a total of 870 ASD patients and 782 controls, suggested an increased risk for ASD in children with vitamin D deficiency. Wang et al. (2021), in a recent publication, continued and updated the initial meta-analysis until November 2019; the results obtained are similar to those already published.

Our study extends the time range until December 2020; on another hand, unlike the aforementioned work, this metaanalysis provides a narrative review of all the selected studies (Table 1) and incorporates data on folic acid levels in children. In this sense, to our knowledge, our study is the most up-to-date meta-analysis carried out on blood levels of vitamin D and folate, measured in groups of children diagnosed with ASD and compared to a group of children without this disorder.

The risk of developing an ASD and its relationship to serum vitamin levels is the main goal of many authors in their studies: cause or consequence? remains a key question in the studies analyzed. We find many authors who argue that a vitamin D deficiency can function as a precursor to the development of ASD: for example, Bener et al. (2017) established that anemia and vitamin D deficiency were more present in the ASD group of participants than in the children of the control group, and proposed that this deficiency is a determinant for this disorder. Similarly, Fernell et al. (2015) stated that low vitamin D levels increase the risk of developing ASD. In this line, Ansary et al. (2018) found a significant inverse association between hypovitaminosis $\mathrm{D}$ and impaired cognitive development and suggested a possible leading role of vitamin D in the development of ASD.

Following the study of the etiology, other authors have focused on analyzing the nutritional status to obtain data on vitamin levels; for instance, Zhu et al. (2020), who observed a higher risk of nutritional deficiencies in the ASD group than in the control group. Petruzzelli et al. (2020) also argued that vitamin D deficiency may pose a risk factor for developing ASD, whereas Gong et al. (2014) went further, proposing that serum levels of $25(\mathrm{OH}) \mathrm{D}$ could be used as an auxiliary diagnostic tool for early detection of ASD.

On another hand, in terms of the cause, following the initial approach, we highlight authors such as Altun et al. (2018) who, in addition to establishing a relationship with vitamin D deficiency and folate with the development of ASD, argue that children with ASD have lower numbers of vitamin receptors, including those of vitamin $\mathrm{D}$, which favors hypovitaminosis.

As can be seen, the study of the causal relationships of ASD, the etiology, symptoms, and course of the disease 
are topics about which there is not yet scientific unanimity. As for vitamin supplementation to correct the deficit, many authors have conducted experimental studies, providing supplements to improve the symptoms of the disease (Bener et al., 2014; Bent et al., 2017; Jia et al., 2015; Song et al., 2020; Wu et al., 2018). Based on the results obtained in our study, we consider that it is necessary to take into account the nutritional status of patients diagnosed with ASD and, therefore, vitamin and fatty acid levels, as these elements are involved in the proper functioning of our body and are key pieces of neurodevelopment.

We could not analyze gender differences in our study given the majority proportion of the male sex in all studies included, some of which included only males in the group of participants diagnosed with ASD (Alzghoul et al., 2020; Bičíková et al., 2019; El-Ansary et al., 2018). According to data provided by the CDC, ASD is diagnosed in all racial, ethnic, and socioeconomic groups, but it is 4.5 times more common in boys than in girls. Similarly, DSM-V (APA., 2014) establishes a 4:1 relationship in favor of male onset.

Moreover, we found three studies that do not establish a relationship between vitamin D and/or folate levels with cases of ASD (Bičíková et al., 2019; Liu et al., 2016; Uğur \& Gürkan, 2014). Causes that might explain these discrepancies with our results could include the sample size and the season of the year when the blood sample was obtained. Thus, we found that in the study of Ugür and Gürkan (2014), they took into account the months of higher and lower sun exposure, unlike the other two studies.

Meta-analyzed data of folate levels in groups of children with ASD showed no significant differences with the controls. However, we found other meta-analyses focused on the association between folate supplementation during pregnancy and the onset of autism (Iglesias et al., 2019; Wang et al., 2017). In the study, Iglesias et al. (2019) found favorable results for the association between reducing the occurrence of new cases of autism, specifically by $58 \%$, with folate supplementation during the prenatal period. Likewise, Wang et al. (2017) achieved similar results, suggesting a reduction in the risk of developing autism in mothers supplemented with folic acid during pregnancy.

We cannot conclude the relationship of fatty acid levels with ASD, as only one study was selected to analyze these levels (Parletta et al., 2016), although there are some indications in this sense. In fact, some studies have carried out fatty acid supplementation interventions, obtaining favorable results for the clinical symptoms of children with ASD, with improved symptoms of irritability, hyperactivity, and social function (Mazahery et al., 2016; Ooi et al., 2015).

Finally, we believe that it is necessary to continue research in this field with larger samples that examine causal relationships for etiological risk factors, as well as works clarifying the causes of vitamin D deficiency in children diagnosed with autism, and the benefits of supplementation.

\section{Conclusions}

The studies contained in this review and the meta-analyzed data allow us to establish the following conclusions, following the proposed objectives.

First, our study has reported vitamin D deficiency in ASD child samples compared to a child control group without this disorder. Likewise, our meta-analysis for this parameter corroborates this fact (Fig. 2).

Regarding blood values of folate and fatty acids, we could not establish significant relationships between a deficit and ASD. More specific studies are needed for these biochemical parameters to indicate these relationships.

\section{Limitations and Strengths}

As in any study of this nature, there is a potential selection bias, which we attempted to avoid by searching and selecting peers as described in the methodological section. The potential publication bias has been taken into account by interpreting the respective funnel plot. On another hand, we found significant heterogeneity among the studies, so subgroup analyses were carried out, the results of which were offered to provide all the necessary information to the reader. However, given the scarcity of studies included in some cases, the results should be considered with caution.

\section{Implications for Future Research and Clinical Practice}

The results indicate the importance of studying vitamin $\mathrm{D}$ and folate supplementation in the treatment of autism. In fact, numerous clinical trials whose main intervention was vitamin D supplementation in children with ASD obtained positive results in the symptomatic improvement of autism. However, there are no direct comparisons for many of these interventions. In this regard, the need for network meta-analyses to assess the overall effect size of these treatments and to establish a hierarchy among them in terms of their effectiveness should be considered. Moreover, some of the studies did not provide data on means and standard deviations or did not use control groups. Similarly, most studies evaluated results regarding vitamin 
D levels, and to a lesser extent, folate; hardly any studies were found to provide complete data on fatty acids, so they could not be included in the meta-analysis. For subsequent studies, we recommend the use of standard measures and homogeneous instruments, as well as the development of clinical studies that include the complete results of these substances to establish clearer relationships about the roles of vitamin $\mathrm{D}$, folate, and fatty acids with ASD.

Finally, considering that the results presented report lower values of vitamin D in children diagnosed with ASD, it would be pertinent to carry out routine analytical controls of these serum levels in children in whom this disorder is diagnosed or who present compatible symptoms.

Supplementary Information The online version contains supplementary material available at https://doi.org/10.1007/s10803-021-05335-8.

Acknowledgments We would like to thank the University of Murcia for its collaboration during the performance of this study.

Author contributions All authors whose names appear on the submission made substantial contributions to the conception or design of the work; or the acquisition, analysis, or interpretation of data; drafted the work or revised it critically for important intellectual content; approved the version to be published; and agree to be accountable for all aspects of the work in ensuring that questions related to the accuracy or integrity of any part of the work are appropriately investigated and resolved. Material preparation, data collection and analysis were performed by Maria Carmen Gallardo-Carrasco and José Antonio Jiménez Barbero. The first draft of the manuscript was written by Maria Carmen Gallardo-Carrasco and all authors commented on previous versions of the manuscript. All authors read and approved the final manuscript. Given the study's methodological peculiarities, it is not necessary to get approval from the ethics committee. So, the manuscript is a retrospective case report and it doesn't need to get approval from the ethics committee.

Funding Open Access funding provided thanks to the CRUE-CSIC agreement with Springer Nature.

\section{Declarations}

Conflict of interest The authors state that they have no potential conflict of interest concerning the investigation, authorship, and/or publication of this article.

Open Access This article is licensed under a Creative Commons Attribution 4.0 International License, which permits use, sharing, adaptation, distribution and reproduction in any medium or format, as long as you give appropriate credit to the original author(s) and the source, provide a link to the Creative Commons licence, and indicate if changes were made. The images or other third party material in this article are included in the article's Creative Commons licence, unless indicated otherwise in a credit line to the material. If material is not included in the article's Creative Commons licence and your intended use is not permitted by statutory regulation or exceeds the permitted use, you will need to obtain permission directly from the copyright holder. To view a copy of this licence, visit http://creativecommons.org/licenses/by/4.0/.

\section{References}

Agostoni, C., Nobile, M., Ciappolino, V., Delvecchio, G., Tesei, A., Turolo, S., Crippa, A., Mazzocchi, A., Altamura, C., \& Brambilla, P. (2017). The role of omega-3 fatty acids in developmental psychopathology: A systematic review on early psychosis, autism, and ADHD. International Journal of Molecular Sciences, 18(12), 1-25. https://doi.org/10.3390/ijms 18122608

Altun, H., Kurutaş, E., Şahin, N., Göngör, O., \& Findikli, E. (2018). The levels of Vitamin D, Vitamin D receptor, homocysteine and complex B Vitamin in children with autism spectrum disorders. Clinical Psychopharmacology and Neuroscience, 16(4), 383-390. https://doi.org/10.9758/cpn.2018.16.1.383

Alzghoul, L., AL-Eitan, L., Aladawi, M., Odeh, M., \& Abu Hantash, O. (2020). The association between serum vitamin D3 levels and autism among Jordanian boys. Journal of Autism and Developmental Disorders, 50(9), 3149-3154. https://doi.org/10.1007/ s10803-019-04017-w

American Psychiatric Association-APA. (2014). Manual Diagnóstico y Estadístico de los trastornos mentales DSM-5. Editorial Médica Panamericana.

Arastoo, A., Khojastehkia, H., Rahimi, Z., Khafaie, M., Hosseini, S., Mansoori, S., Mohammad, T., Yosefyshad, S., Abshirini, M., Karimimalekabadi, N., \& Cheraghi, M. (2018). Evaluation of serum 25-Hydroxy vitamin D levels in children with autism Spectrum disorder. Italian Journal of Pediatrics, 44(1), 1-5. https://doi.org/10.1186/s13052-018-0587-5

Bala, K. A., Doğan, M., Kaba, S., Mutluer, T., Aslan, O., \& Doğan, S. Z. (2016). Hormone disorder and vitamin deficiency in attention deficit hyperactivity disorder (ADHD) and autism spectrum disorders (ASDs). Journal of Pediatric Endocrinology \& Metabolism: JPEM, 29(9), 1077-1082.

Belinchón, M., Boada, L., García de Andrés, E., Fuentes, J., \& Posada de la Paz, M. (2010). Evolución de los estudios sobre autismo en España: Publicaciones y redes de coautoría entre 1974 y 2007. Psicothema, 22(2), 242-249.

Bener, A., Khattab, A., \& Al-Dabbagh, M. (2014). Is high prevalence of Vitamin D deficiency evidence for autism disorder?: In a highly endogamous population. Journal of Pediatric Neurosciences, 9(3), 227-233. https://doi.org/10.4103/1817-1745. 147574

Bener, A., Khattab, A., Bhugra, D., \& Hoffmann, G. (2017). Iron and Vitamin D levels among autism spectrum disorders children. Annals of African Medicine, 16(4), 186-191. https://doi.org/10. 4103/aam.aam_17_17

Bent, S., Ailarov, A., Dang, K., Widjaja, F., Lawton, B., \& Hendren, R. (2017). Open-label trial of vitamin D3 supplementation in children with autism spectrum disorder. Journal of Alternative and Complementary Medicine, 23(5), 394-395. https://doi.org/ 10.1089/acm.2016.0297

Bičíková, M., Máčová, L., Ostatníková, D., \& Hanzlíková, L. (2019). Vitamin D in Autistic children and healthy controls. Physiological Research, 68(2), 317-320. https://doi.org/10.33549/physiolres. 933902

Booth, A., Clarke, M., Dooley, G., Ghersi, D., Moher, D., Petticrew, M., \& Stewart, L. (2013). PROSPERO at one year: an evaluation of its utility. Systematic Reviews, 2(4), 1-7. https://doi.org/ 10.1186/2046-4053-2-4

Bozzatello, P., Brignolo, E., De Grandi, E., \& Bellino, S. (2016). Supplementation with omega-3 fatty acids in psychiatric disorders: A review of literature data. Journal of Clinical Medicine, 5(8), 1-29. https://doi.org/10.1016/B978-1-893997-82-0.50004-9

Cermak, S. A., Curtin, C., \& Bandini, L. G. (2010). Food selectivity and sensory sensitivity in children with autism spectrum 
disorders. Journal of the American Dietetic Association, 110(2), 238-246. https://doi.org/10.1016/j.jada.2009.10.032

Chen, L., Shi, X., Liu, H., Mao, X., Gui, L., Wang, H., \& Cheng, Y. (2021). Oxidative stress marker aberrations in children with autism spectrum disorder: A systematic review and meta-analysis of 87 studies $(\mathrm{N}=9109)$. Translational Psychiatry. https://doi. org/10.1038/s41398-020-01135-3

De Luca, H. (2004). Overview of general physiologic features and functions of vitamin D. The American Journal of Clinical Nutrition, 80(6 Suppl), 1689-1696. https://doi.org/10.1093/ajen/80.6. $1689 \mathrm{~s}$

Du, L., Shan, L., Wang, B., Feng, J., Xu, Z., \& Jia, F. (2015). Serum levels of 25-hydroxyvitamin $\mathrm{D}$ in children with autism spectrum disorders. Chinese Journal of Contemporary Pediatrics, 17(1), 68-71. https://doi.org/10.7499/j.issn.1008-8830.2015.01.015

El-Ansary, A., Cannell, J., Bjørklund, G., Bhat, R., Al Dbass, A., Alfawaz, H., Chirumbolo, S., \& Al-Ayadhi, L. (2018). In the search for reliable biomarkers for the early diagnosis of autism spectrum disorder: The role of vitamin D. Metabolic Brain Disease, 33(3), 917-931. https://doi.org/10.1007/s11011-018-0199-1

Esteban-Figuerola, P., Canals, J., Fernández-Cao, J., \& Arija-Val, V. (2019). Differences in food consumption and nutritional intake between children with autism spectrum disorders and typically developing children: A meta-analysis. Autism, 23(5), 1079-1095. https://doi.org/10.1177/1362361318794179

Eyles, D., Burne, T., \& McGrathMcGrath, J. (2013). Vitamin D, effects on brain development, adult brain function and the links between low levels of vitamin $\mathrm{D}$ and neuropsychiatric disease. Frontiers in Neuroendocrinology, 34, 47-64.

Farid, S., El-hamamsy, M., \& Zaki, O. (2016). Vitamin d intake and sun exposure in autistic children. International Journal of Pharmaceutical Sciences and Research, 7(3), 1043-1049. https://doi. org/10.13040/IJPSR.0975-8232.7(3).1043-49

Fernell, E., Bejerot, S., Westerlund, J., Miniscalco, C., Simila, H., Eyles, D., Gillberg, C., \& Humble, M. (2015). Autism spectrum disorder and low vitamin D at birth: A sibling control study. Molecular Autism, 6(1), 1-9. https://doi.org/10.1186/ 2040-2392-6-3

Frye, R., Slattery, J., Delhey, L., Furgerson, B., Strickland, T., Tippett, M., Sailey, A., Wynne, R., Rose, S., Melnyk, S., Jill James, S., Sequeira, J. M., \& Quadros, E. V. (2018). Folinic acid improves verbal communication in children with autism and language impairment: A randomized double-blind placebocontrolled trial. Molecular Psychiatry, 23(2), 247-256. https:// doi.org/10.1038/mp.2016.168

Fuentes, J., Basurko, A., Isasa, I., Galende, I., Muguerza, M., GarcíaPrimo, P., García, J., Fernández-Álvarez, C., Canal-Bedia, R., $\&$ Posada de la Paz, M. (2020). The ASDEU autism prevalence study in northern Spain. European Child and Adolescent Psychiatry. https://doi.org/10.1007/s00787-020-01539-y

Garipardic, M., Doğan, M., Bala, K., Mutluer, T., Kaba, S., Aslan, O., \& Üstyol, L. (2017). Association of attention deficit hyperactivity disorder and autism spectrum disorders with mean platelet volume and vitamin D. Medical Science Monitor, 23, 1378-1384. https://doi.org/10.12659/MSM.899976

Gong, Z., Luo, C., Wang, L., Shen, L., Wei, F., Tong, R., \& Liu, Y. (2014). Serum 25-hydroxyvitamin D levels in Chinese children with autism spectrum disorders. NeuroReport, 25(1), 23-27. https://doi.org/10.1097/WNR.0000000000000034

Guo, M., Li, L., Zhang, Q., Chen, L., Dai, Y., Liu, L., Feng, J., Cai, X., Cheng, Q., Chen, J., Wei, H., \& Li, T. (2020). Vitamin and mineral status of children with autism spectrum disorder in Hainan Province of China: Associations with symptoms. Nutritional Neuroscience, 23(10), 803-810. https://doi.org/10.1080/ 1028415X.2018.1558762
Guo, M., Zhu, J., Yang, T., Lai, X., Lei, Y., Chen, J., \& Li, T. (2019). Vitamin A and vitamin D deficiencies exacerbate symptoms in children with autism spectrum disorders. Nutritional Neuroscience, 22(9), 637-647. https://doi.org/10.1080/1028415X.2017. 1423268

Holick, M. F. (2017). The vitamin D deficiency pandemic: Approaches for diagnosis, treatment and prevention. Reviews in Endocrine \& Metabolic Disorders, 25, 153-165. https://doi. org/10.1007/s11154-017-9424-1

Iglesias, L., Canals, J., \& Arija-Val, V. (2019). Review and metaanalysis found that prenatal folic acid was associated with a $58 \%$ reduction in autism but had no effect on mental and motor development. Acta Paediatrica, International Journal of Paediatrics, 108(4), 600-610. https://doi.org/10.1111/apa.14657

Jia, F., Wang, B., Shan, L., Xu, Z., Staal, W., \& Du, L. (2015). Core symptoms of autism improved after vitamin D supplementation. Pediatrics, 135(1), 196-198.

Kanner, L. (1943). Autistic disturbances of affective contact. Nervous Child, 2(3), 217-250.

Kerub, O., Haas, E., Meiri, G., Bilenko, N., Flusser, H., Michaelovski, A., Dinstein, I., Davidovitch, N., \& Menashe, I. (2021). Ethnic disparities in the diagnosis of autism in Southern Israel. Autism Research, 14(1), 193-201. https://doi.org/ 10.1002/aur.2421

Kheirouri, S., \& Alizadeh, M. (2020). Maternal excessive gestational weight gain as a risk factor for autism spectrum disorder in offspring: A systematic review. BMC Pregnancy and Childbirth, 20(1), 1-12. https://doi.org/10.1186/s12884-020-03324-w

Kinney, D., Barch, D., Chayka, B., Napoleon, S., \& Munir, K. (2010). Environmental risk factors for autism: Do they help cause de novo genetic mutations that contribute to the disorder? Medical Hypotheses, 74(1), 102-106. https://doi.org/10.1016/j.mehy.2009.07.052

Liu, D., Zhan, J., \& Shao, J. (2015). Environmental risk factors for autism spectrum disorders in children. Chinese Journal of Contemporary Pediatrics, 17(11), 1147-1153. https://doi.org/10. 7499/j.issn.1008-8830.2015.11.001

Liu, X., Liu, J., Xiong, X., Yang, T., Hou, N., Liang, X., Chen, J., Cheng, Q., \& Li, T. (2016). Correlation between nutrition and symptoms: Nutritional survey of children with autism spectrum disorder in Chongqing, China. Nutrients, 8(5), 1-15. https://doi. org/10.3390/nu8050294

Lord, C., Elsabbagh, M., Baird, G., \& Veenstra-Vanderweele, J. (2018). Autism spectrum disorder. The Lancet, 392(10146), 508-520. https://doi.org/10.1016/S0140-6736(18)31129-2

Maenner, M. J., Shaw, K., Baio, J., Washington, A., Patrick, M., DiRienzo, M., Christensen, D., Wiggins, L., Pettygrove, S., Andrews, J., Lopez, M., Hudson, A., Baroud, T., Schwenk, Y., White, T., Rosenberg, C., Lee, L., Harrington, R., Huston, M., ... Dietz, P. (2020). Prevalence of autism spectrum disorder among children aged 8 Years-Autism and developmental disabilities monitoring network, 11 Sites, United States, 2016. MMWR Surveillance Summaries, 69(4), 1-12. https://doi.org/10.15585/MMWR.SS6904A1

Málaga, I., Lago, R., Hedrera-Fernández, A., Álvarez-álvarez, N., Oreña-Ansonera, V., \& Baeza-Velasco, M. (2019). Prevalence of autism spectrum disorders in USA, Europe and Spain: Coincidences and discrepancies. Medicina, 79(1), 4-9.

Marín, F., Alcantud, E., Yurena, A., \& Mata, S. (2016). Prevalencia de los trastornos del espectro autista: revisión de datos. Universidad de Salamanca.

Matson, J. L., \& Kozlowski, A. M. (2011). The increasing prevalence of autism spectrum disorders. Research in Autism Spectrum Disorders, 5(1), 418-425. https://doi.org/10.1016/j.rasd.2010.06.004

Mazahery, H., Conlon, C., Beck, K., Kruger, M., Stonehouse, W., Camargo, C., Meyer, B., Tsang, B., Mugridge, O., \& von Hurst, P. (2016). Vitamin D and omega-3 fatty acid supplements in children with autism spectrum disorder: A study protocol for a factorial 
randomised, double-blind, placebo-controlled trial. Trials, 17(1), 1-13. https://doi.org/10.1186/s13063-016-1428-8

Moher, D., Shamseer, L., Clarke, M., Ghersi, D., Liberati, A., Petticrew, M., Shekelle, P., \& Stewart, L. A. (2015). Preferred reporting items for systematic review and meta-analysis protocols (PRISMA-P) 2015 statement. Systematic Reviews, 4(1), 148-160. https://doi.org/10.1186/2046-4053-4-1

Morales-Hidalgo, P., Roigé-Castellví, J., Hernández-Martínez, C., Voltas, N., \& Canals, J. (2018). Prevalence and characteristics of autism spectrum disorder among spanish school-age children. Journal of Autism and Developmental Disorders, 48(9), 31763190. https://doi.org/10.1007/s10803-018-3581-2

Nevison, C., \& Parker, W. (2020). California autism prevalence by county and race/ethnicity: declining trends among wealthy whites. Journal of Autism and Developmental Disorders, 50(11), 40114021. https://doi.org/10.1007/s10803-020-04460-0

Ogbu, D., Xia, E., \& Sun, J. (2020). Gut instincts: Vitamin D/vitamin $D$ receptor and microbiome in neurodevelopment disorders: VDR and microbiome in gut-brain axis. Open Biology. https://doi.org/ 10.1098/rsob.200063rsob200063

Ooi, Y., Weng, S., Jang, L., Low, L., Seah, J., Teo, S., Ang, R., Lim, C., Liew, A., Fung, D., \& Sung, M. (2015). Omega-3 fatty acids in the management of autism spectrum disorders: Findings from an open-label pilot study in Singapore. European Journal of Clinical Nutrition, 69(8), 969-971. https://doi.org/10.1038/ejcn.2015.28

Page, M., McKenzie, J., Bossuyt, P., Boutron, I., Hoffmann, T., Mulrow, C., Shamseer, L., Tetzlaff, J., Akl, E., Brennan, S., Chou, R., Glanville, J., Grimshaw, J., Hróbjartsson, A., Lalu, M., Li, T., Loder, E., Mayo-Wilson, E., McDonald, S., ... Moher, D. (2021). The PRISMA 2020 statement: An updated guideline for reporting systematic reviews. Systematic Reviews, 10(1). https://doi.org/10. 1186/s13643-021-01626-4

Parletta, N., Niyonsenga, T., \& Duff, J. (2016). Omega-3 and omega-6 polyunsaturated fatty acid levels and correlations with symptoms in children with attention deficit hyperactivity disorder, autistic spectrum disorder and typically developing controls. PLOS ONE, 11(5), 1-17. https://doi.org/10.1371/journal.pone.0156432

Petruzzelli, M., Marzulli, L., Margari, F., De Giacomo, A., Gabellone, A., Giannico, O., \& Margari, L. (2020). Vitamin D deficiency in autism spectrum disorder: A cross-sectional study. Disease Markers. https://doi.org/10.1155/2020/9292560

Sánchez, S. S., Lorente, A., Pineda, O., Fernández-Cao, J. C., \& ArijaVal, V. (2015). Selectividad alimentaria en los trastornos del espectro autista: Una revisión sistemática. Revista Española De Nutrición Comunitaria, 21, 13-19.

Sánchez-Meca, J., \& Marín-Martínez, F. (2010). Meta-analysis in psychological research. International Journal of Psychological Research, 3(1), 150-162.

Şengenç, E., Kıykım, E., \& Saltik, S. (2020). Vitamin D levels in children and adolescents with autism. Journal of International Medical Research. https://doi.org/10.1177/0300060520934638

Song, L., Luo, X., Jiang, Q., Chen, Z., Zhou, L., Wang, D., \& Chen, A. (2020). Vitamin D supplementation is beneficial for children with autism spectrum disorder: A meta-analysis. Clinical Psychopharmacology and Neuroscience, 18(2), 203-213. https://doi.org/10. 9758/cpn.2020.18.2.203

Sun, C., Zou, M., Zhao, D., Xia, W., \& Wu, L. (2016). Efficacy of folic acid supplementation in autistic children participating in structured teaching: An open-label trial. Nutrients, 8(6), 1-14. https://doi.org/10.3390/nu8060337

Thorsen, M., Bilenberg, N., Thorsen, L., \& Michel, T. (2021). Oxidative stress in adults with autism spectrum disorder: A case control study. Journal of Autism and Developmental Disorders, Harrison. https://doi.org/10.1007/s10803-021-04897-x

Tioleco, N., Silberman, A. E., Stratigos, K., Banerjee-Basu, S., Spann, M. N., Whitaker, A. H., \& Turner, J. B. (2021). Prenatal maternal infection and risk for autism in offspring: A meta-analysis. Autism Research. https://doi.org/10.1002/aur.2499

Uğur, Ç., \& Gürkan, C. K. (2014). Serum vitamin D and folate levels in children with autism spectrum disorders. Research in Autism Spectrum Disorders, 8(12), 1641-1647.

Von Elm, E., Altman, D., Egger, M., Pocock, S., Gøtzsche, P., \& Vandenbroucke, J. (2007). The strengthening the reporting of observational studies in epidemiology (STROBE) statement: Guidelines for reporting observational studies. PLoS Medicine, 4(10), 1623-1627. https://doi.org/10.1371/journal.pmed.0040296

Wang, M., Li, K., Zhao, D., \& Li, L. (2017). The association between maternal use of folic acid supplements during pregnancy and risk of autism spectrum disorders in children: A meta-analysis. Molecular Autism, 8(1), 4-7. https://doi.org/10.1186/ s13229-017-0170-8

Wang, T., Shan, L., Du, L., Feng, J., Xu, Z., Staal, W. G., \& Jia, F. (2016). Serum concentration of 25- hydroxyvitamin D in autism spectrum disorder: A systematic review and meta-analysis. European Child \& Adolescent Psychiatry, 25(4), 341-350.

Wang, Z., Ding, R., \& Wang, J. (2021). The association between vitamin d status and autism spectrum disorder (Asd): A systematic review and meta-analysis. Nutrients, 13(1), 1-14. https://doi.org/ 10.3390/nu13010086

Wu, D., Wen, X., Han, X., Wang, S., Wang, Y., Shen, M., Fan, S., Zhuang, J., Li, M., Hu, B., Sun, C., Bao, Y., Yan, J., Lu, J., \& Zheng, Y. (2018). Relationship between neonatal vitamin D at birth and risk of autism spectrum disorders: The NBSIB study. Journal of Bone and Mineral Research, 33(3), 458-466. https:// doi.org/10.1002/jbmr.3326

Yule, S., Wanik, J., Holm, E., Bruder, M., Shanley, E., Sherman, C., Fitterman, M., Lerner, J., Marcello, M., Parenchuck, N., Romanwhite, C., \& Ziff, M. (2020). Nutritional deficiency disease secondary to ARFID symptoms associated with autism and the broad autism phenotype: A qualitative systematic review of case reports and case series. Journal of the Academy of Nutrition and Dietetics. https://doi.org/10.1016/j.jand.2020.10.017

Zhu, J., Guo, M., Yang, T., Lai, X., Tang, T., Chen, J., Li, L., \& Li, T. (2020). Nutritional status and symptoms in preschool children with autism spectrum disorder: A two-center comparative study in Chongqing and Hainan Province, China. Frontiers in Pediatrics. https://doi.org/10.3389/fped.2020.00469

Publisher's Note Springer Nature remains neutral with regard to jurisdictional claims in published maps and institutional affiliations. 